\title{
Efficient generation of hepatocyte-like cells from human induced pluripotent stem cells
}

\author{
Zhihua Song ${ }^{1,2}$, Jun Cai ${ }^{1}$, Yanxia Liu ${ }^{1}$, Dongxin Zhao ${ }^{1}$, Jun Yong ${ }^{1,2}$, Shuguang Duo ${ }^{1}$, Xijun Song ${ }^{1}$, Yushan Guo ${ }^{1,2}$, \\ Yang Zhao ${ }^{1}$, Han Qin ${ }^{1,2}$, Xiaolei Yin ${ }^{1,2}$, Chen $\mathrm{Wu}^{1,2}$, Jie Che ${ }^{1}$, Shichun $\mathrm{Lu}^{3}$, Mingxiao Ding ${ }^{1}$, Hongkui Deng ${ }^{1,2}$ \\ ${ }^{I}$ The MOE Key Laboratory of Cell Proliferation and Differentiation, Department of cell biology, College of Life Sciences, Box 38, \\ Peking University, Beijing 100871, China; ${ }^{2}$ Laboratory of Chemical Genomics, Shenzhen Graduate School of Peking University, \\ Shenzhen 518055, China; ${ }^{3}$ Beijing YouAn Hospital, Capital Medical University, Beijing 100069, China
}

Human induced pluripotent stem (iPS) cells are similar to embryonic stem (ES) cells, and can proliferate intensively and differentiate into a variety of cell types. However, the hepatic differentiation of human iPS cells has not yet been reported. In this report, human iPS cells were induced to differentiate into hepatic cells by a stepwise protocol. The expression of liver cell markers and liver-related functions of the human iPS cell-derived cells were monitored and compared with that of differentiated human ES cells and primary human hepatocytes. Approximately $60 \%$ of the differentiated human iPS cells at day 7 expressed hepatic markers alpha fetoprotein and Alb. The differentiated cells at day 21 exhibited liver cell functions including albumin Asecretion, glycogen synthesis, urea production and inducible cytochrome P450 activity. The expression of hepatic markers and liver-related functions of the iPS cellderived hepatic cells were comparable to that of the human ES cell-derived hepatic cells. These results show that human iPS cells, which are similar to human ES cells, can be efficiently induced to differentiate into hepatocyte-like cells.

Keywords: induced pluripotent stem cells, iPS, differentiation, hepatic cells, embryonic stem cells Cell Research (2009) 19:1233-1242. doi: 10.1038/cr.2009.107; published online 8 September 2009

\section{Introduction}

Hepatocyte transplantation restores damaged liver

Correspondence: Hongkui Deng

Fax: +86-010-6275-6954

E-mail: hongkui_deng@p ku.edu.cn

Abbreviations: iPS cells (induced pluripotent stem cells); ES cells (embryonic stem cells); MEF (mouse embryonic fibroblast); HCM (Hepatocyte Culture Medium); FGF4 (fibroblast growth factor 4); BMP2 (bone morphogenetic protein 2); HGF (hepatocyte growth factor); KGF/FGF7 (keratinocyte growth factor/fibroblast growth factor 7); OCT4 (octamerbinding factor 4); KLF4 (Krüppel-like factor4); UTF1 (undifferentiated embryonic cell transcription factor 1); PBS (phosphate-buffered saline); SOX (SRY-box containing gene); FoxA2 (forkhead box A2); CK (cytokeratin); AFP (alpha fetoprotein); ALB (albumin); AAT (alpha 1-antitrypsin); Cyp (cytochrome P450); PEPCK (phosphoenolpyruvate carboxykinase); HNF $4 \alpha$ (hepatocyte nuclear factor $4 \alpha$ ); HNF6 (hepatocyte nuclear factor 6); CEBP $\alpha$ (CCAAT enhancer binding protein alpha); TDO2 (tryptophan 2,3-dioxygenase); RT-PCR (reverse transcription PCR); PAS (Periodic Acid-Schiff)

Received 26 June 2009; revised 21 July 2009; accepted 21 July 2009; published online 8 September 2009 function in host animals and serves as a promising way to replace orthotopic liver transplantation for the treatment of a wide range of liver diseases [1]. However, sources of donor human hepatocytes are limited, and patients who receive a liver transplant require immune suppressive drugs. Embryonic stem (ES) cells essentially proliferate infinitely in vitro and maintain the ability to differentiate into a variety of tissue cells, including hepatocytes. Thus, ES cells can serve as an inexhaustible cell source for hepatocyte transplantation [2].

Induced pluripotent stem (iPS) cells were generated directly from fibroblast cells as the result of the introduction of four factors [3-7], and shared many characteristics with ES cells, including multi-lineage differentiation potential and intensive proliferation in vitro. In addition, the establishment of human iPS cells is ethically acceptable and does not require oocytes. Thus, through human iPS cell differentiation, genetically identical somatic cells could be generated for the treatment of a wide variety of diseases. The human iPS cells were shown to be able to differentiate into neural cells [8-11], osteogenic cells 
[12], cardiac cells [13], adipogenic cells [14], pancreatic cells $[15,16]$, vascular cells [17], hematopoietic $[12,18]$ and endothelial cells [18]. However, the directed differentiation of human iPS cells into hepatic cells has not yet been reported.

We have recently developed an efficient method to establish human iPS cell lines with a new set of four transcription factors [19], and we have previously established a protocol to induce the efficient hepatic differentiation of human ES cells [20]. In this study, human iPS cell lines were induced to differentiate into hepatic cells with a modified protocol. The differentiated iPS cells expressed hepatic markers and possessed human hepatocyte functions. By monitoring the expression of liver cell markers at different differentiation stages, we found that human iPS cells can be directly induced to differentiate into hepatocyte-like cells with the very similar differentiation process to that of human ES cells. Our study shows that human iPS cells could potentially be a promising cell source for liver disease-related researches.

\section{Results}

Characterization of human iPS cells

In this study, we utilized human iPS cell lines 3U1 and
$3 \mathrm{U} 2$ as two representative cell lines from a panel of human iPS cell lines that were generated in our laboratory. These two cell lines were established by transducing the genes Oct-4, Sox2, Klf4 and Utf1 into adult human fibroblasts [19]. To check the stemness of the iPS cell lines, the expression of core transcription factors and surface markers including octamer-binding factor 4 (OCT4), NANOG, SRY-box containing gene (SOX)2, SSEA4, TRA1-60 and TRA1-81 was tested by reverse transcription PCR (RT-PCR) or immunofluorescence assay, and the results showed that the expression pattern of these stem cell markers in iPS cells is similar to that of the H1 hES cells (Supplementary information, Figure S1).

\section{Efficient hepatic differentiation of human iPS cells}

Here, we modified our previous protocol [20] and developed an improved differentiation method for hES cells and human iPS cells, as shown in Figure 1A. This new hepatic differentiation protocol was composed of four stages: endoderm induction, hepatic specification, hepatoblast expansion and hepatic maturation. 3U1 and 3U2 iPS cells were able to differentiate into hepatic cells in comparison to a well-established human ES cell line H1, which was able to be induced efficiently to differentiate into hepatic lineage cells [20]. During the differentiation,

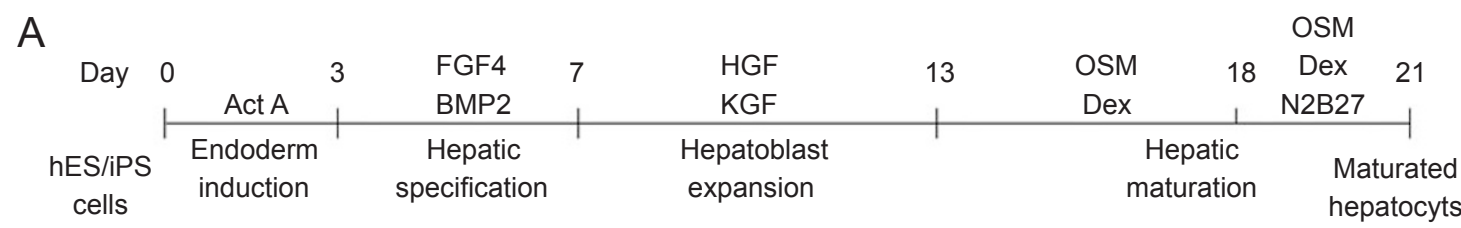

B

Day 0

Day 3

Day 7

Day 21
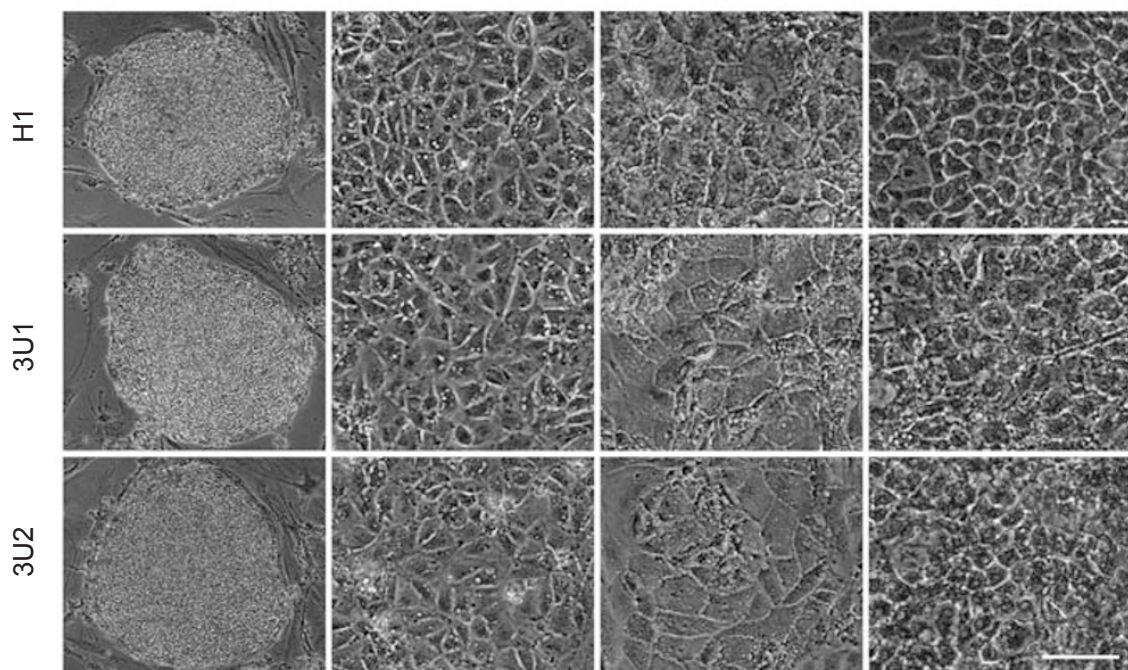

Figure 1 The hepatic differentiation of human iPS cells and ES cells. (A) A flow chart showing the stepwise differentiation protocol. (B) Morphological changes of human ES cells and iPS cells at different stages of differentiation. Scale bar $=100 \mu \mathrm{m}$. 
A
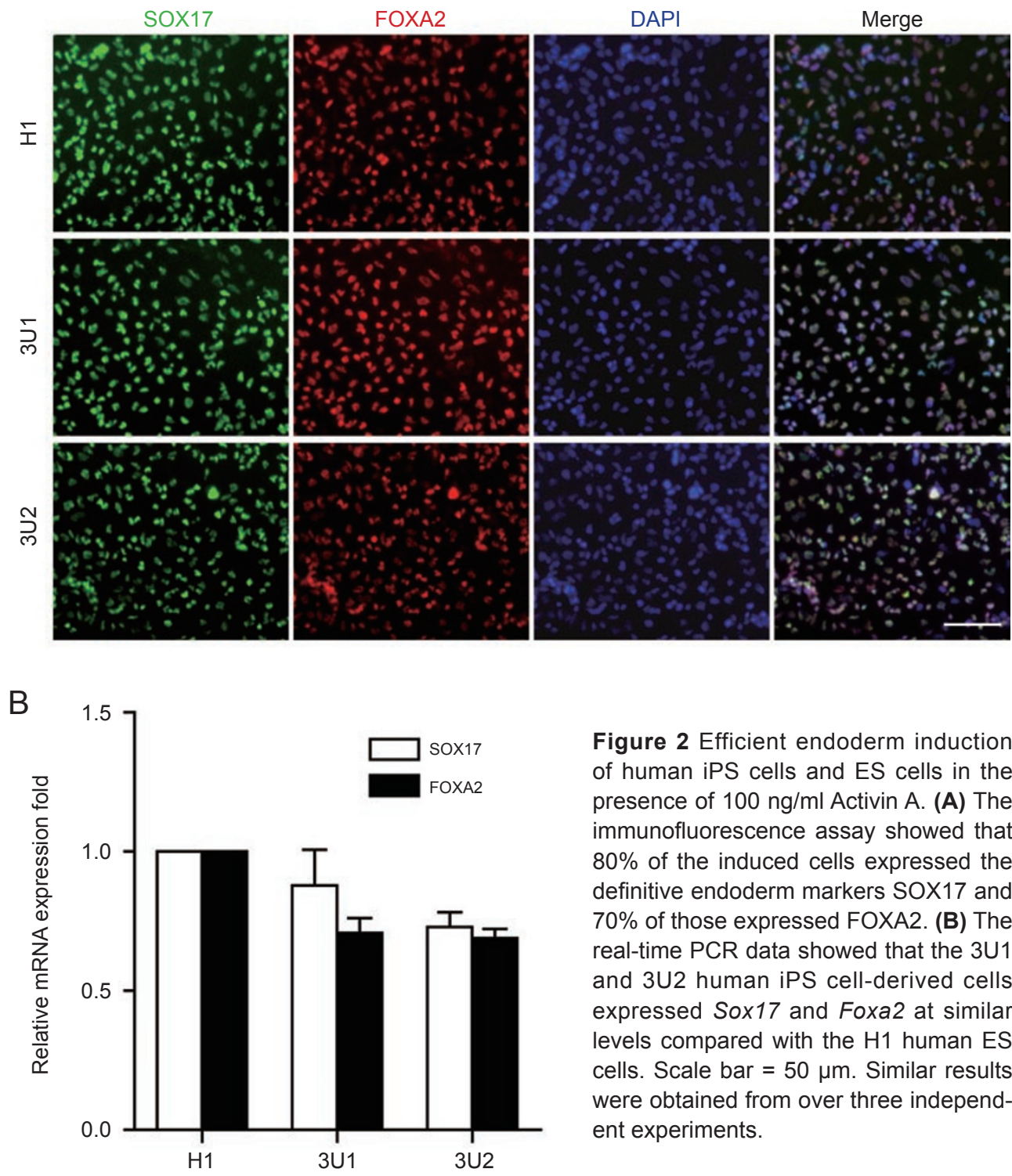

Figure 2 Efficient endoderm induction of human iPS cells and ES cells in the presence of $100 \mathrm{ng} / \mathrm{ml}$ Activin A. (A) The immunofluorescence assay showed that $80 \%$ of the induced cells expressed the definitive endoderm markers SOX17 and $70 \%$ of those expressed FOXA2. (B) The real-time $\mathrm{PCR}$ data showed that the $3 \mathrm{U} 1$ and $3 \mathrm{U} 2$ human iPS cell-derived cells expressed Sox17 and Foxa2 at similar levels compared with the $\mathrm{H} 1$ human ES cells. Scale bar $=50 \mu \mathrm{m}$. Similar results were obtained from over three independent experiments.

the cell morphology change of 3U1 and 3U2 human iPS cells resembled that of the H1 hES cells at each differentiation stage (Figure 1B).

In the first stage, Activin A efficiently induced the endoderm differentiation of both human iPS cell lines $3 \mathrm{U} 1$ and $3 \mathrm{U} 2$, and the hES cell line H1. After 3 days of Activin A treatment, approximately $80 \%$ of the cells in culture expressed the endoderm marker SOX17, 70\% of the cells in culture expressed another endoderm marker forkhead box A2 (FOXA2), and about $50-60 \%$ of the total cells in culture were double positive (Figure 2A), but no cell was found to express the extraembryonic endoderm cell markers SOX7 or CDX2 (data not shown), as detected by immune staining of the differentiated cells.
The expression of endoderm genes in the differentiated cells was also checked by real-time PCR, and the results showed that human iPS cell-derived cells express endoderm markers SOX17 and FOXA2 at similar levels compared with H1 hES cell-derived cells (Figure 2B), while at day 0 , neither hES cells $\mathrm{H} 1$ nor iPS cells $3 \mathrm{U} 1$ and $3 \mathrm{U} 2$ expressed SOX17 or FOXA2 (Supplementary information, Figure S2A and S2B). Similar results were obtained from over three independent experiments. These results indicate that human iPS cells could be efficiently induced to differentiate into endoderm cells by Activin A.

To induce hepatic specification from endoderm cells, fibroblast growth factor 4 (FGF4) and bone morphogenetic protein 2 (BMP2) were added to the culture. After 
A

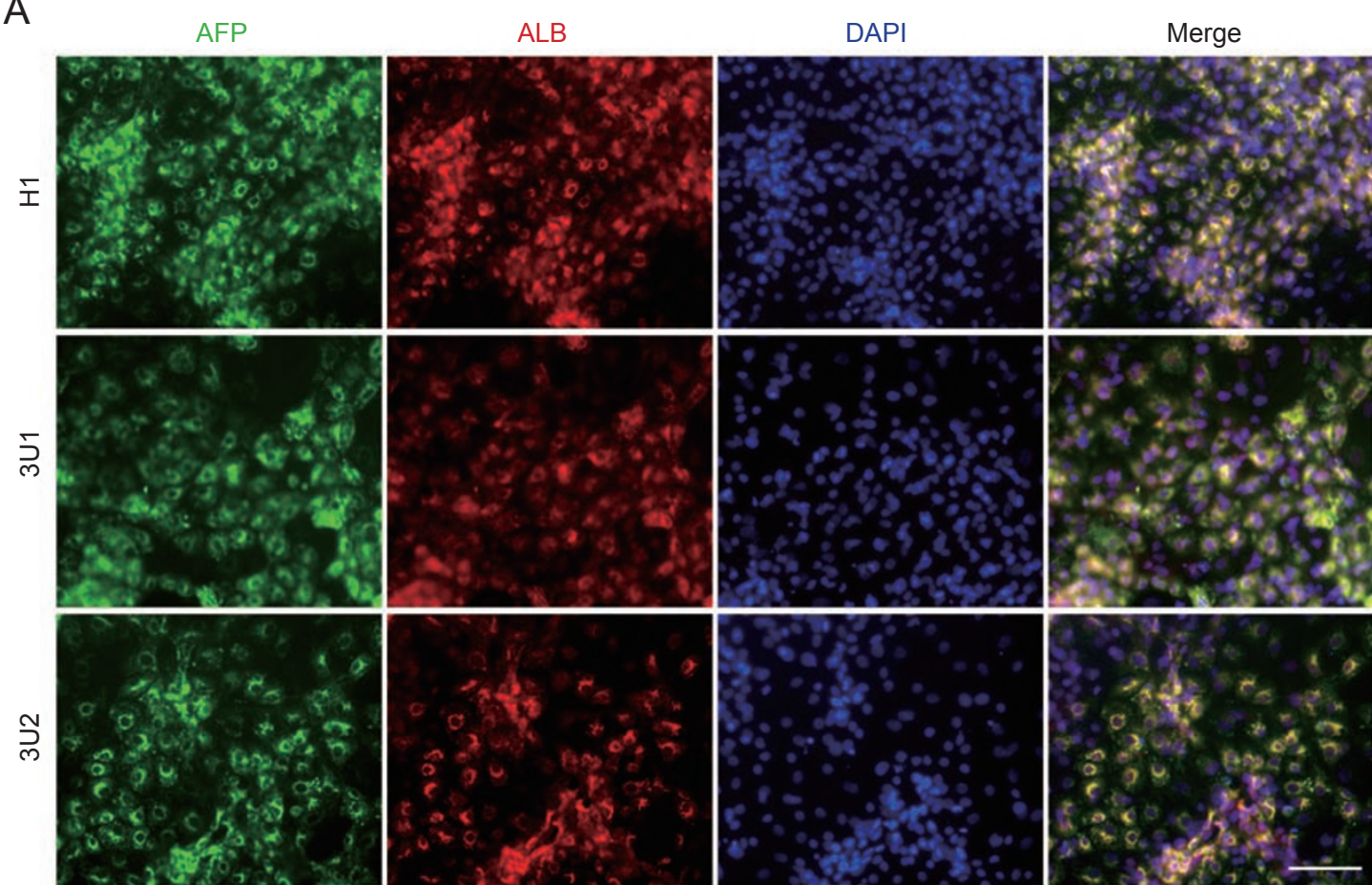

B

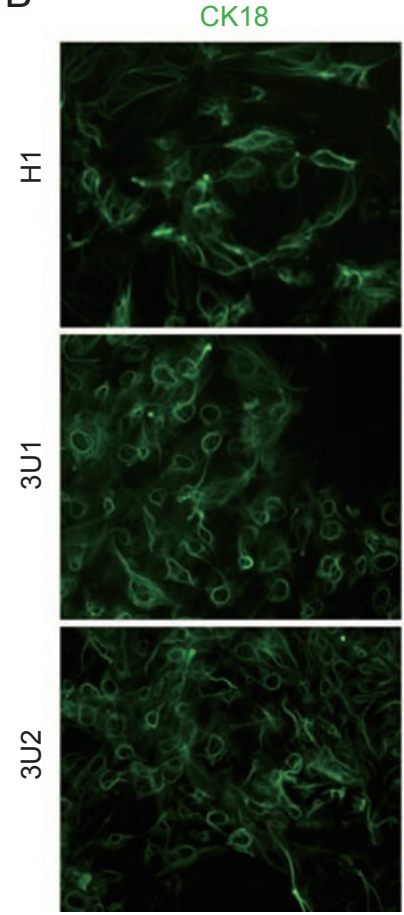

DAPI

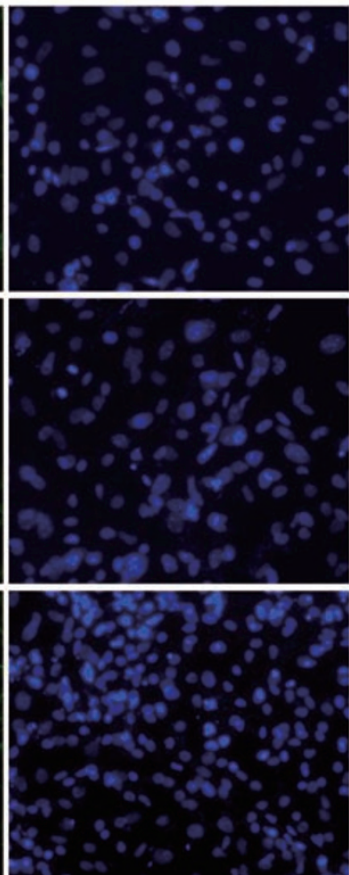

Merge

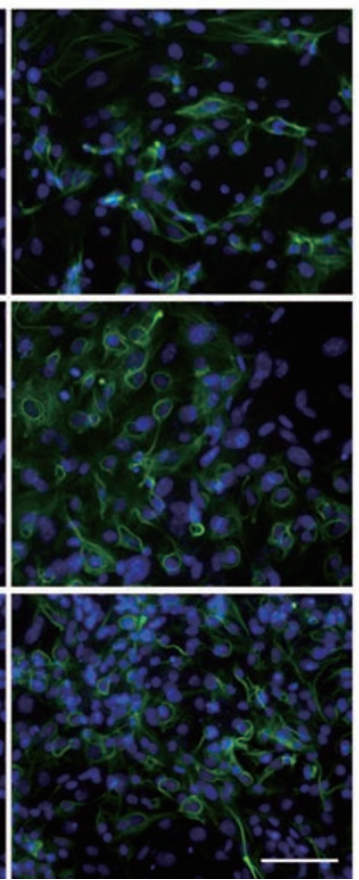

C

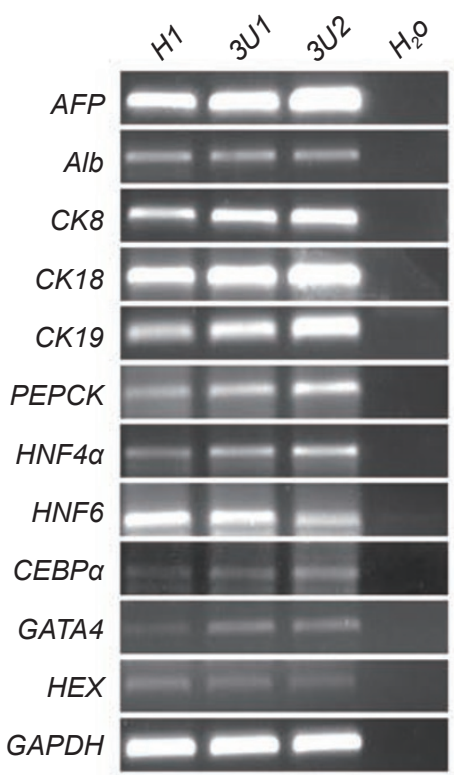

Figure 3 Hepatic specification of human iPS cell- and ES cell-derived endoderm cells after 4 days of treatment with FGF and BMP. (A, B) At day 7, the differentiated cells expressed the hepatic cell markers AFP, ALB and CK18. (C) AFP, Alb, CK8, CK18, CK19 and PEPCK mRNA were expressed at this stage and the liver-enriched transcription factors $H N F 4 \alpha, H N F 6$, $C E B P \alpha$, GATA4 and $H E X$ were activated. $\mathrm{H}_{2} \mathrm{O}$ was used as a negative control. Scale bar $=100 \mu \mathrm{m}$. Similar results were obtained in at least three independent experiments. 
4 days of treatment, the cells were tested for the expression of hepatic markers and liver-enriched transcription factors by immunostaining or RT-PCR. For both iPS cell and hES cell differentiation, approximately $60 \%$ of the cells in culture expressed the hepatic cell markers alpha fetoprotein (AFP), ALB (albumin) (Figure 3A) and more

A

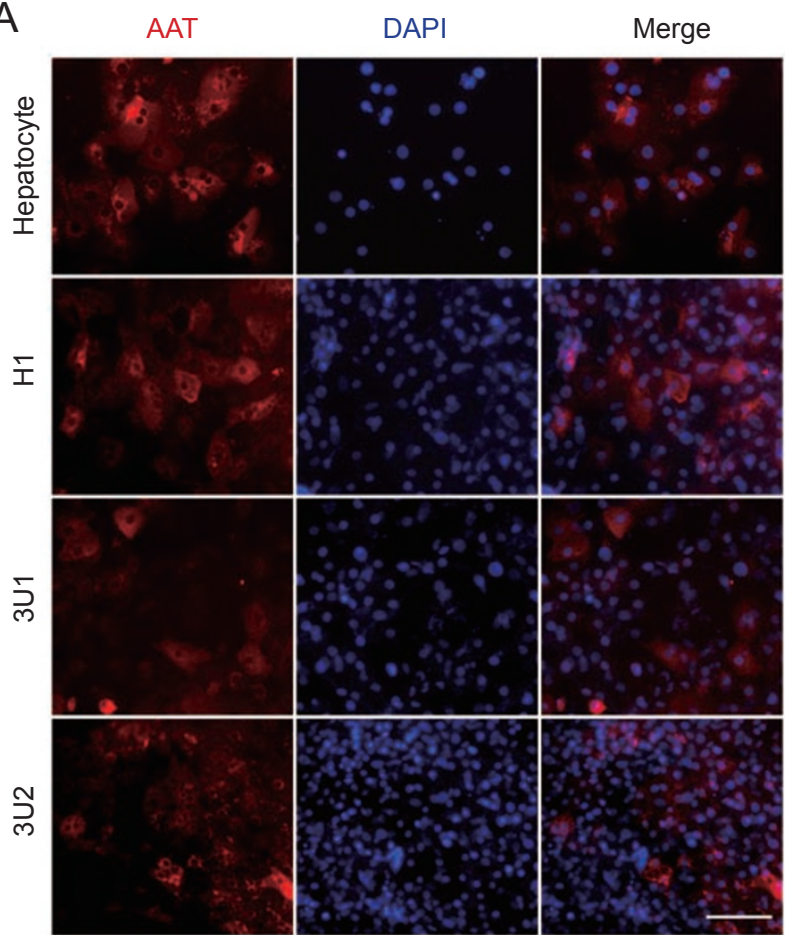

C

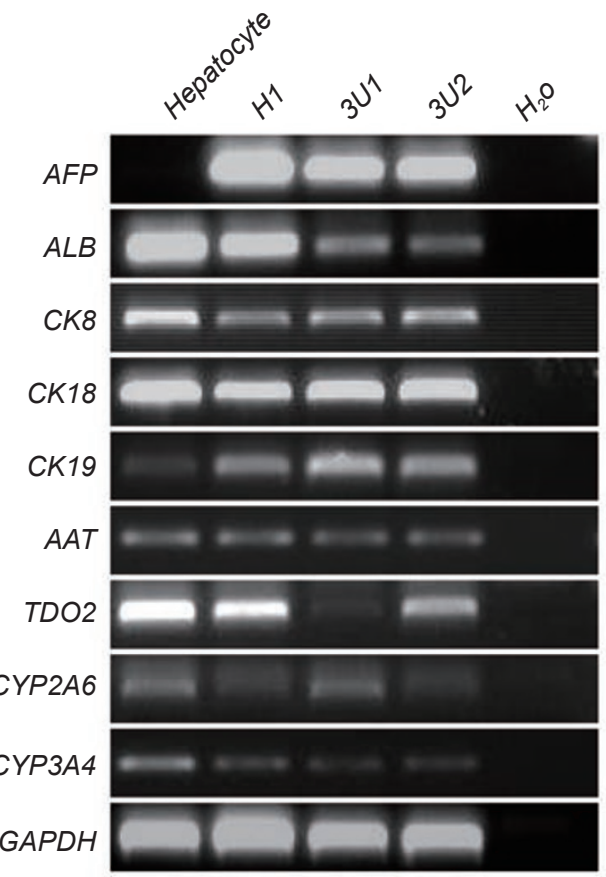

differentiated cells expressed cytokeratin (CK)18 (Figure 3B), as detected by immunofluorescence. We checked the expression of AFP, ALB and CK18 at day 0 in the undifferentiated iPS cells and found negative staining (Supplementary information, Figure S2). The differentiated cells also expressed hepatic genes including $A F P$,

B

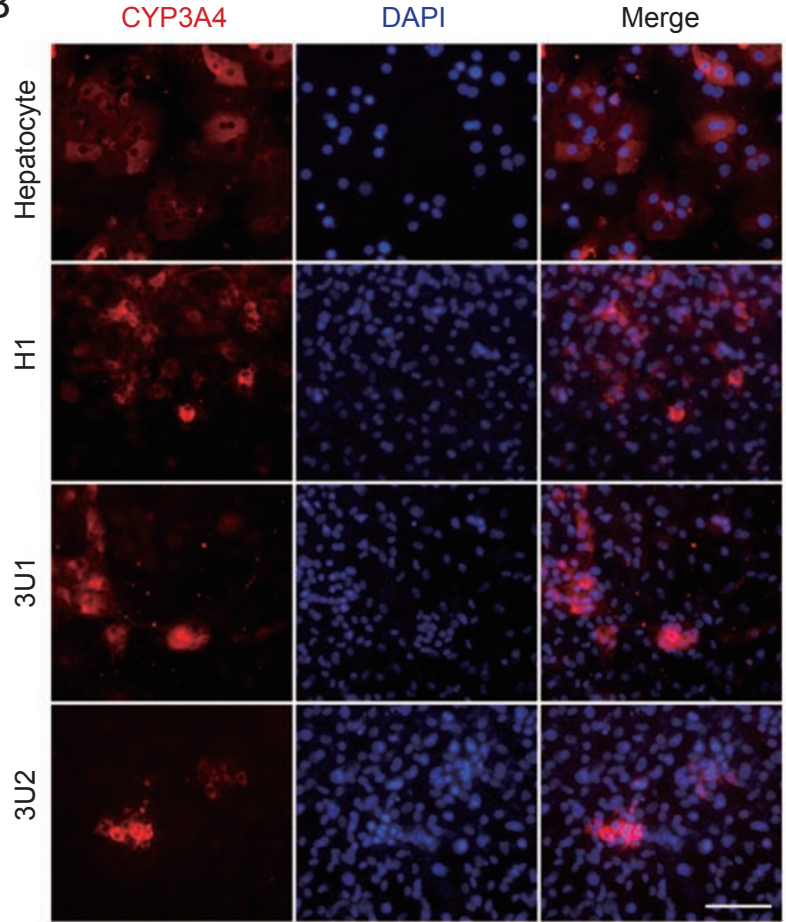

Figure 4 Hepatic maturation of differentiated human iPS cells and ES cells. (A, B) The differentiated cells possessed markers indicative of functional hepatocytes, including AAT and CYP3A4 at day 21. (C) The expression of AFP, Alb, CK8, CK18, CK19, AAT, TDO2, CYP2A6 and CYP3A4 of day-21 differentiated cells was detected by RT-PCR. Human hepatocytes and $\mathrm{H}_{2} \mathrm{O}$ were used as positive and negative controls, respectively. Scale bar $=100 \mu \mathrm{m}$. Similar results were obtained in at least three independent experiments. 
A
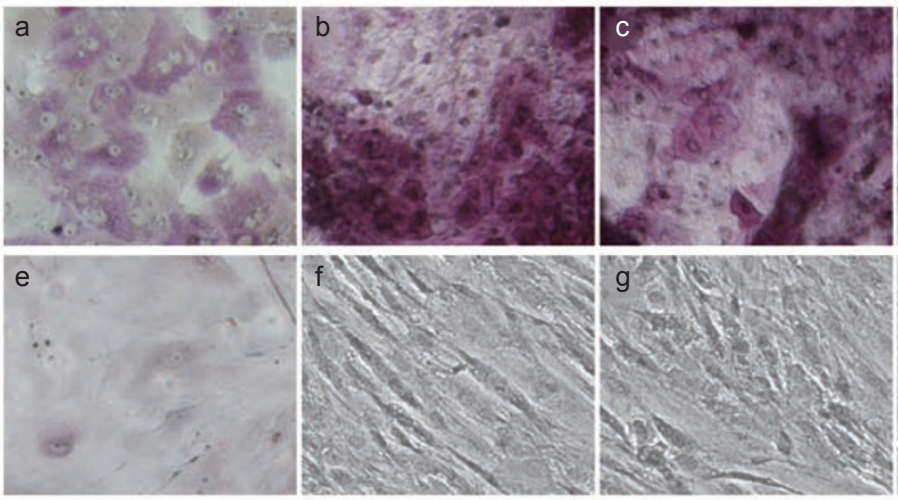
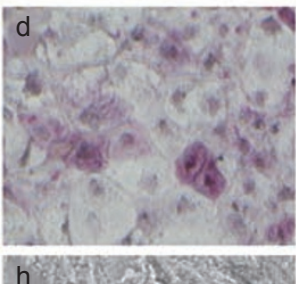

B

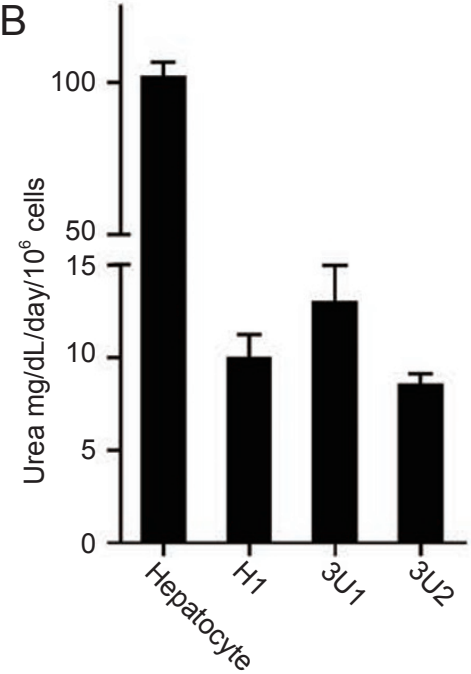

C

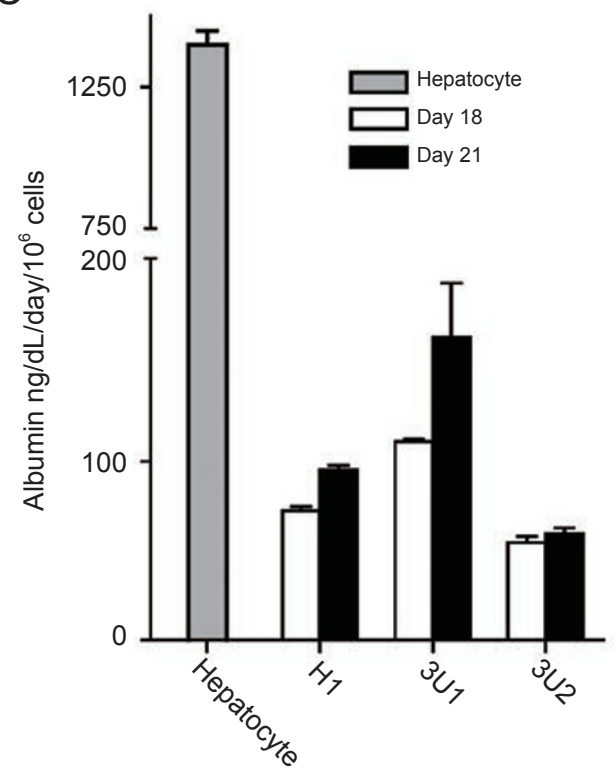

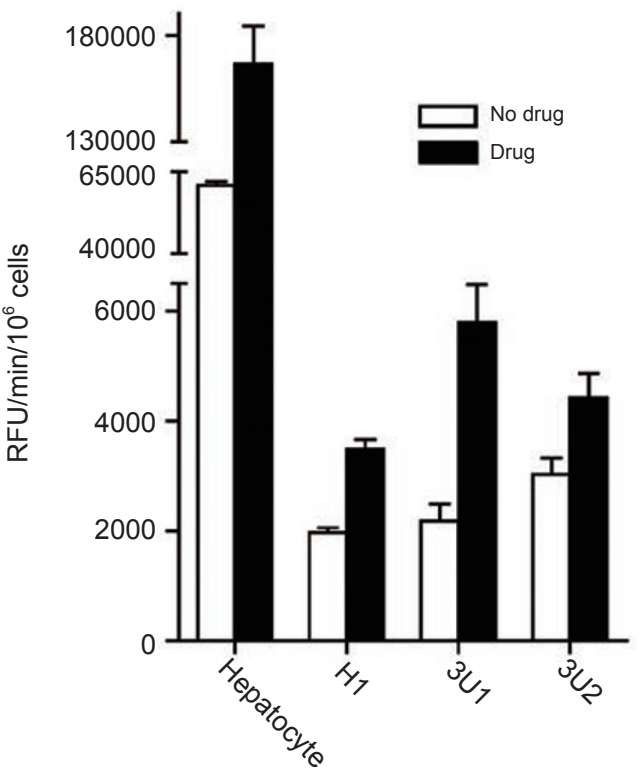

Figure 5 Functional testing of the maturated hepatic cells derived from human iPS cells and ES cells. (A) Glycogen synthesis was tested by PAS staining at day 21. a and e, human hepatocytes and feeder cells, respectively; b, c, d and f, g, h represent $\mathrm{H} 1$ ES cell-, 3U1 and 3U2 iPS cell derivatives with or without growth factors, respectively. Scale bar $=100 \mu \mathrm{m}$. $(\mathrm{B}) \mathrm{Urea}$ productions by H1-, 3U1- and 3U2-derived cells. (C) Albumin secretion of differentiated cells from H1 ES cell-, 3U1 and $3 U 2$ iPS cells. (D) The differentiated human iPS cells and hES cells exhibited cytochrome P450 isozyme activities after phenobarbital sodium induction. Human hepatocytes were used as a positive control.

$A L B, C K 8, C K 18, C K 19$ and PEPCK and the liverenriched transcription factors $H N F 4 \alpha, H N F 6, C E B P \alpha$, GATA4 and HEX at the mRNA level, as detected by RTPCR (Figure 3C), while these genes were not expressed in the undifferentiated iPS cells and hES cells (data not shown). These results indicated that human iPS cells could be efficiently induced into hepatic lineage at this stage. Similar results were obtained in at least three independent experiments.

To expand these early hepatic cells, hepatocyte growth factor (HGF) and keratinocyte growth factor (KGF) were then added to the culture medium during the third stage. We tested for the expression of Ki67, a marker of proliferating cells, at day 13 in the differentiated $\mathrm{H} 1 \mathrm{hES}$ cells and $3 \mathrm{U} 1$ and 3U2 iPS cells. As shown in Supplementary information, Figure S3, compared to the control group that did not have KGF treatment, the addition of KGF led to more Ki67-positive cells in culture, and consequently more cells were double positive for AFP and Ki67. Similar results were obtained in at least three independent 
experiments.

At the end of the final differentiation stage, we tested the expression of hepatic markers in the differentiated $3 \mathrm{U} 1$ and $3 \mathrm{U} 2$ human iPS cells. A majority of the day21 cells expressed hepatic markers, such as AFP and Alb (data not shown). Furthermore, at day 21, some of the differentiated 3U1 and 3U2 human iPS cells were stained positive for alpha 1-antitrypsin (AAT) (Figure 4A) and CYP3A4 (Figure 4B), a pattern similar to H1 hES cell derivatives and human hepatocytes. The RT-PCR data showed that the hepatic functional genes, including $A l b$, $A A T, T D O 2, C Y P 2 A 6$ and $C Y P 3 A 4$, as well as other liver-enriched genes including $A F P, C K 8, C K 18$ and $C K 19$ were expressed (Figure $4 C$ ). Similar results were obtained in at least three independent experiments.

\section{Evaluation of the maturated hepatic cells derived from human iPS cells}

We performed several experiments to further test liver cell-specific functions in the differentiated human iPS cells. At day 21, we assayed glycogen storage by Periodic Acid-Schiff (PAS) staining in the hepatic cells derived from the 3U1 and 3U2 human iPS cells with amylase pre-treatment. Similar to H1 hES cell-derived cells and human hepatocytes, many of the differentiated $3 \mathrm{U} 1$ and 3U2 human iPS cells were stained by PAS (Figure $5 \mathrm{~A})$. The differentiated cells of both H1 hES cells and $3 \mathrm{U} 1$ and $3 \mathrm{U} 2$ iPS cells without growth factor treatment showed fibroblast-like morphology, and were hardly PAS positive (Figure $5 \mathrm{~A}, \mathrm{f}, \mathrm{g}$ and $\mathrm{h}$ ). In addition, the mouse embryonic fibroblast (MEF) feeder cells were also PAS negative (Figure 5A, e).

Urea synthesis of the differentiated cells was tested with a urea nitrogen determination system. At the end of the hepatic maturation stage, urea could be detected in the culture medium supernatant of the day 21 differentiated $3 \mathrm{U} 1$ and $3 \mathrm{U} 2$ human iPS cells. The production levels were similar to that of $\mathrm{hES}$ cell derivatives (Figure $5 \mathrm{~B})$. We performed this assay thrice with four parallel samples in each experiment, and similar results were obtained. Human hepatocytes were used here as a positive control.

To detect albumin secretion, we collected culture medium supernatant samples and measured albumin levels with an ELISA assay. Hepatic cells derived from 3U1 and 3U2 human iPS cells secreted albumin at the similar level to hES cell derivatives both at days 18 and 21 (Figure $5 \mathrm{C})$. The culture medium of undifferentiated iPS cells and hES cells including MEF feeder had a blank readout in the ELISA assay test. We repeated this assay thrice with four parallel samples each time, and obtained similar results. Human hepatocytes were used as a positive control.

The inducible P450 activity of the differentiated cells was also tested. As shown in Figure 5D, the differentiated human iPS cells exhibited cytochrome P450 isozyme activities after phenobarbital sodium induction, and the pattern of response to the drug induction was similar to hES cell-differentiated cells (Figure 5D). We performed this assay thrice with two parallel samples with or without drug induction in each experiment, and similar results were obtained. Human hepatocytes were used here as a positive control. Altogether, these functional results indicate that the differentiated human iPS cells are similar to human ES cell derivatives, and that the induced hepatocyte-like cells from human iPS cells possess some characteristics of adult hepatocytes.

\section{Discussion}

Here, we show that human iPS cells can be directly induced to differentiate into hepatocyte-like cells using a stepwise differentiation method. The hepatic differentiation efficiency of human iPS cells is comparable to that of the H1 human ES cell line. Our results showed that during human iPS cell differentiation, the process of endoderm cell induction and hepatocyte marker expression was very similar to that of differentiated human ES cells. The functions and hepatic marker expression of the human iPS cell-derived hepatic cells were similar to that of the hepatic cells differentiated from human ES cells (Figures 4, 5 and Supplementary information, Table S1). To our knowledge, this is the first time that human iPS cells are shown to be able to be directly differentiated into hepatic cells. The iPS cell technology allows for the generation of patient-specific iPS cells. Therefore, by comparing the hepatic differentiation process of iPS cells from liver disease patients and that of normal iPS cells, the in vitro differentiation of human iPS cells could serve as a model to study the mechanisms of human liver disease development.

Some previous reports studying iPS cell differentiation showed that the gene expression patterns differ between the cells differentiated from iPS and ES cells [14, 21]. However, the iPS cells used in these studies were established in the presence of c-Myc. Because c-Myc is essential for the self-renewal and differentiation of ES cells $[22,23]$, the reactivation of c-Myc may interfere with the differentiation of iPS cells. To build a disease model with iPS cells, it is preferable to use iPS cell lines established without c-Myc. In the future, it would be better to use human iPS cell lines established by non-integrative methods [24-28].

An important potential use of human iPS cell-derived 
hepatic cells is for drug development. Most drugs rely on liver P450 activity for detoxification, which cannot be tested in animal liver cells due to species differences [29]. CYP3A4 is involved in the metabolism of a large range of xenobiotics. In this study, we tested the expression of CYP in the differentiated cells and found that the human iPS cell- and ES cell-derived hepatic cells expressed Cyp3A4, as detected by RT-PCR (Figure 4C) and immune staining (Figure 4B). These cells also possessed inducible P450 2B4 activity (Figure 5D), suggesting that human iPS cell-derived hepatic cells possess liver cell functions in certain extent and may also be used as a potential cell source for the generation of hepatocytes for drug metabolism tests. Because human iPS cells can be established from different populations, human iPS cellderived hepatic cells could potentially be used to study the differences in drug metabolism among populations.

In this study, we compared human ES cell- and iPS cell-derived hepatic cells with human hepatocytes in liver-enriched gene expression and liver-specific functions (Figures 4, 5 and Supplementary information, Table S1). Considering the urea synthesis and albumin secretion, the production levels of either hES cell- or iPS cell-derived hepatic cells were approximately $10 \%$ of those of human hepatocytes (Figure 5B and 5C). The cytochrome P450 isozyme activities of the differentiated hES and iPS cells were nearly 30 -fold lower than those of human hepatocytes either at basal level or after drug induction (Figure 5D). In addition, the liver-enriched genes including $A A T$, TDO2, CYP2A6 and CYP3A4 examined in the differentiated hES and iPS cells were less expressed compared with human hepatocytes (Figure 4C). These results suggest that the differentiated human iPS cells and ES cells need to be further improved at the final maturation stage.

\section{Materials and Methods}

Culture and differentiation of the human iPS cells and ES cells into definitive endoderm

The human ES cell line H1 was obtained from the WiCell research institute (Madison, WI, USA). The passage number of the H1 cells used here ranged from 33 to 48. Human iPS cell lines $3 \mathrm{U} 1$ and $3 \mathrm{U} 2$ were established by transducing human fibroblasts with lentivirus containing Oct-4, Sox2, Klf4 and Utfl, as previously described [19]. The passage number of the $3 \mathrm{U} 1$ and $3 \mathrm{U} 2$ cells used here ranged from 4 to 19. Human ES cells and iPS cells were cultured in DMEM/F12 with $20 \%$ knockout serum replacement, $1 \mathrm{mM} / 1$ glutamax, $1 \%$ nonessential amino acids, 0.1 $\mathrm{mM} \beta$-mercaptoethanol, $100 \mathrm{U}$ penicillin/streptomycin (all from Invitrogen/Gibco, Grand Island, NY, USA), and $10 \mathrm{ng} / \mathrm{ml}$ basic fibroblast growth factor (Peprotech, Rocky Hill, NJ, USA) on a mitomycin-C-treated MEF feeder layer in a standard gas atmosphere of humid air with $5 \% \mathrm{CO}_{2}$.

For endoderm induction, human ES cells and iPS cells were incubated for $24 \mathrm{~h}$ with RPMI 1640 medium (Invitrogen/Gibco, Rockville, MD, USA), supplemented with $0.5 \mathrm{mg} / \mathrm{ml}$ albumin fraction V (Sigma-Aldrich, St Louis, MO, USA) and $100 \mathrm{ng} /$ $\mathrm{ml}$ Activin A (Peprotech). On the following 2 days, 0.1 and $1 \%$ insulin-transferrin-selenium (Invitrogen/Gibco) was added to this medium, respectively.

\section{Hepatic differentiation of human iPS cells and ES cells}

Following Activin A treatment, the differentiated human iPS cells and hES cells were cultured in Hepatocyte Culture Medium (HCM) (Cambrex, Baltimore, MD, USA) containing $30 \mathrm{ng} / \mathrm{ml}$ FGF4 and $20 \mathrm{ng} / \mathrm{ml} \mathrm{BMP} 2$ (both from Peprotech) for 4 days. Then, the differentiated cells were incubated in HCM containing $20 \mathrm{ng} /$ $\mathrm{ml} \mathrm{HGF}$ and $20 \mathrm{ng} / \mathrm{ml} \mathrm{KGF}$ (both from Peprotech) for 6 days, in HCM containing $10 \mathrm{ng} / \mathrm{ml}$ oncostatin-M (R\&D, Minneapolis, MN, USA) plus $0.1 \mu \mathrm{M}$ dexamethasone (Sigma-Aldrich) for 5 days, and in DMEM containing N2, B27, 1 mM/1 glutamax, $1 \%$ nonessential amino acids and $0.1 \mathrm{mM} \beta$-mercaptoethanol (all from Invitrogen/ Gibco) for another 3 days.

\section{Human hepatocytes isolation and culture}

Primary human hepatocytes were isolated from donor livers that were not used for liver transplantation following informed consent, by a two-step EDTA/collagenase perfusion and then cryopreserved [30]. Cell viability was more than $80 \%$ as determined by trypan blue exclusion. After thawing, human hepatocytes were cultured in matrigel (BD, NJ, USA) coated dishes with HCM. The medium was refreshed everyday.

\section{Immunofluorescence assay}

Cells were fixed with 4\% paraformaldehyde (Dingguo, Beijing, China) for $15 \mathrm{~min}$, blocked and permeabilized with $10 \%$ normal goat or rabbit serum (Zsbio, Beijing, China) and $0.2 \%$ tritonX-100 (Fisher, UK) in phosphate-buffered saline (PBS) at room temperature for $45 \mathrm{~min}$. Then the cells were incubated with primary antibody overnight at $4{ }^{\circ} \mathrm{C}$. For surface markers staining, the permeabilization step was omitted. Cells were washed with PBS between each step. The primary antibodies against human rabbit anti Oct4 (Abcam, La Jolla, CA, USA), goat anti Nanog (R\&D), mouse anti SSEA4, mouse anti TRA-1-60, mouse anti TRA-1-81 (Santa Cruz, CA), rabbit anti Ki67 (Invitrogen), goat anti SOX17 (R\&D), rabbit anti FOXA2 (Upstate), mouse anti CK18 and mouse anti AFP (Invitrogen) were diluted at 1:200, the antibody against human rabbit anti ALB (DAKO, Glostrup, Denmark) was diluted at 1:500, and the antibodies against human rabbit anti AAT (Invitrogen) and rabbit anti CYP3A4 (AbD Serotec, Oxford, UK) were diluted at 1:200. After five washes with PBS, FITC-conjugated or TRITC-conjugated secondary antibody (Invitrogen) was diluted at $1: 200$ and applied to the cells at $4{ }^{\circ} \mathrm{C}$ overnight. Then, $1 \mu \mathrm{g} / \mathrm{ml}$ 4,6-diamidino-2-phenylindole (Roche, Germany) was used to stain the cell nuclei. Also, corresponding isotype antibody or the normal serum from the same species with the primary antibody was used as negative control.

\section{RT-PCR and real-time PCR analysis of gene expression}

Reverse transcription PCR and real-time PCR were performed as described previously [20]. Total RNA was isolated from cells using TRIzol Reagent (Invitrogen) and reverse-transcribed using a reverse transcription system (Promega, Madison, WI, USA) ac- 
cording to the manufacturer's protocol. Prior to reverse transcription, the RNA samples were treated with RNase-free DNase I (NEB, Ipswich, MA, USA) to remove the trace DNA contamination.

\section{PAS stain for glycogen}

The PAS staining system was purchased from Sigma-Aldrich. Cells in culture dishes were fixed in $4 \%$ paraformaldehyde and were treated with $5 \mathrm{~g} / 1$ amylase (Sigma) for $15 \mathrm{~min}$ at $37{ }^{\circ} \mathrm{C}$ as described [31]. The further assay was performed according to the manufacturer's instructions.

\section{Urea nitrogen kinetic quantitative determination}

The urea nitrogen determination system was purchased from STANBIO (Boerne, TX, USA). Cells were trypsinized and counted with a hemocytometer. The sample supernatants were stored at $-20{ }^{\circ} \mathrm{C}$ and the assay was performed according to the manufacturer's instructions. We measured the absorbance using a Synergy HT Multi-Detection Microplate Reader from BioTek with Gen5 software. The urea production was normalized to the total cell numbers.

\section{Albumin secretion ELISA assay}

The human albumin content in the supernatant was determined by the Human Albumin ELISA Quantitation kit (Bethyl Laboratory, Montgomery, TX, USA) according to the manufacturer's instructions. Cells were trypsinized and counted with a hemocytometer. The albumin secretion was normalized to the total cell numbers.

\section{Cytochrome P450 activity assay}

The cytochrome P450 2B fluorescent detection kit was purchased from Sigma-Aldrich. For the cytochrome P450 activity assay, $200 \mu \mathrm{g} / \mathrm{ml}$ phenobarbital sodium was added to the differentiated human iPS and ES cells during the last 3 days and human hepatocytes for 3 days. The medium was refreshed everyday. The samples were homogenized with an Ultrasonic crusher (Scientz, Ningbo, China) and the assay was performed according to the manufacturer's instructions. We measured the fluorescence with the BioTek Multi-Detection Microplate Reader, using Gen5 software. The maximum kinetic reaction velocity was normalized to the total cell numbers.

\section{Acknowledgments}

We thank Dr Zicai Liang and Huang Huang (Institute of Molecular Medicine, Peking University) for their kind help with BioTek Multi-Detection Microplate Reader and Yizhe Zhang for technical support on real-time PCR. We also thank Chengyan Wang, Pengbo Zhang, Pingping Hou, Haisong Liu, Chun Liu and other colleagues in our laboratory for technical assistance and advice in carrying out these experiments. This study was supported by a Bill \& Melinda Gates Foundation Grant (37871), a Ministry of Education grant (705001), the National Basic Research Program of China (973 program, 2009CB522502, 2009CB941200 and 2007CB947901), National Natural Science Foundation of China for Creative Research Groups (30421004), the Chinese Science and Technology Key Project (2008zx10002-014, 2008zx10002011 and 2009ZX10004-403) and a 111 Project to Deng H.

\section{References}

1 Enns GM, Millan MT. Cell-based therapies for metabolic liver disease. Mol Genet Metab 2008; 95:3-10.

2 Haridass D, Narain N, Ott M. Hepatocyte transplantation: waiting for stem cells. Curr Opin Organ Transplant 2008; 13:627-632.

3 Park IH, Zhao R, West JA, et al. Reprogramming of human somatic cells to pluripotency with defined factors. Nature 2008; 451:141-146.

4 Lowry WE, Richter L, Yachechko R, et al. Generation of human induced pluripotent stem cells from dermal fibroblasts. Proc Natl Acad Sci USA 2008; 105:2883-2888.

5 Yu J, Vodyanik MA, Smuga-Otto K, et al. Induced pluripotent stem cell lines derived from human somatic cells. Science 2007; 318:1917-1920.

6 Takahashi K, Tanabe K, Ohnuki M, et al. Induction of pluripotent stem cells from adult human fibroblasts by defined factors. Cell 2007; 131:861-872.

7 Takahashi K, Yamanaka S. Induction of pluripotent stem cells from mouse embryonic and adult fibroblast cultures by defined factors. Cell 2006; 126:663-676.

8 Dimos JT, Rodolfa KT, Niakan KK, et al. Induced pluripotent stem cells generated from patients with ALS can be differentiated into motor neurons. Science 2008; 321:1218-1221.

9 Chambers SM, Fasano CA, Papapetrou EP, et al. Highly efficient neural conversion of human ES and iPS cells by dual inhibition of SMAD signaling. Nat Biotechnol 2009; 27:275280.

10 Karumbayaram S, Novitch BG, Patterson M, et al. Directed differentiation of human-induced pluripotent stem cells generates active motor neurons. Stem Cells 2009; 27:806-811.

11 Hirami Y, Osakada F, Takahashi K, et al. Generation of retinal cells from mouse and human induced pluripotent stem cells. Neurosci Lett 2009; 458:126-131.

12 Karner E, Unger C, Cerny R, et al. Differentiation of human embryonic stem cells into osteogenic or hematopoietic lineages: a dose-dependent effect of osterix over-expression. $J$ Cell Physiol 2009; 218:323-333.

13 Zhang J, Wilson GF, Soerens AG, et al. Functional cardiomyocytes derived from human induced pluripotent stem cells. Circ Res 2009; 104:e30-e41.

14 Taura D, Noguchi M, Sone M, et al. Adipogenic differentiation of human induced pluripotent stem cells: comparison with that of human embryonic stem cells. FEBS Lett 2009; 583:1029-1033.

15 Tateishi K, He J, Taranova O, et al. Generation of insulinsecreting islet-like clusters from human skin fibroblasts. $J$ Biol Chem 2008; 283:31601-31607.

16 Zhang D, Jiang W, Liu M, et al. Highly efficient differentiation of human ES cells and iPS cells into mature pancreatic insulin-producing cells. Cell Res 2009; 19:429-438.

17 Taura D, Sone M, Homma K, et al. Induction and isolation of vascular cells from human-induced pluripotent stem cells. Arterioscler Thromb Vasc Biol 2009; 29:1100-1103.

18 Choi KD, Yu J, Smuga-Otto K, et al. Hematopoietic and endothelial differentiation of human induced pluripotent stem cells. Stem Cells 2009; 27:559-567. 
19 Zhao Y, Yin X, Qin H, et al. Two supporting factors greatly improve the efficiency of human iPSC generation. Cell Stem Cell 2008; 3:475-479.

20 Cai J, Zhao Y, Liu Y, et al. Directed differentiation of human embryonic stem cells into functional hepatic cells. Hepatology 2007; 45:1229-1239.

21 Mauritz C, Schwanke K, Reppel M, et al. Generation of functional murine cardiac myocytes from induced pluripotent stem cells. Circulation 2008; 118:507-517.

22 Sumi T, Tsuneyoshi N, Nakatsuji N, Suemori H. Apoptosis and differentiation of human embryonic stem cells induced by sustained activation of c-Myc. Oncogene 2007; 26:55645576.

23 Cartwright P, McLean C, Sheppard A, Rivett D, Jones K, Dalton S. LIF/STAT3 controls ES cell self-renewal and pluripotency by a Myc-dependent mechanism. Development 2005; 132:885-896.

$24 \mathrm{Yu}$ J, Hu K, Smuga-Otto K, et al. Human induced pluripotent stem cells free of vector and transgene sequences. Science 2009; 324:797-801.

25 Woltjen K, Michael IP, Mohseni P, et al. piggyBac transposition reprograms fibroblasts to induced pluripotent stem cells.
Nature 2009; 458:766-770.

26 Kaji K, Norrby K, Paca A, Mileikovsky M, Mohseni P, Woltjen K. Virus-free induction of pluripotency and subsequent excision of reprogramming factors. Nature 2009; 458:771-775.

27 Gonzalez F, Barragan Monasterio M, Tiscornia G, et al. Generation of mouse-induced pluripotent stem cells by transient expression of a single nonviral polycistronic vector. Proc Natl Acad Sci USA 2009; 106:8918-8922.

28 Zhou H, Wu S, Joo JY, et al. Generation of induced pluripotent stem cells using recombinant proteins. Cell Stem Cell 2009; 4:381-384.

29 Tuschl G, Lauer B, Mueller SO. Primary hepatocytes as a model to analyze species-specific toxicity and drug metabolism. Expert Opin Drug Metab Toxicol 2008; 4:855-870.

30 Berry MN, Friend DS. High-yield preparation of isolated rat liver parenchymal cells: a biochemical and fine structural study. J Cell Biol 1969; 43:506-520.

31 Campard D, Lysy PA, Najimi M, Sokal EM. Native umbilical cord matrix stem cells express hepatic markers and differentiate into hepatocyte-like cells. Gastroenterology 2008; 134:833-848.

(Supplementary information is linked to the online version of the paper on the Cell Research website.) 